Revue d'histoire de l'Amérique française

सQS REVUE D.HISTOIRE DE L'AMÉRIQUE FRANÇAISE

\title{
Les Français et le Canada, 1850-1914 : identité et perception
}

\section{Sylvain Simard}

Volume 29, numéro 2, septembre 1975

URI : https://id.erudit.org/iderudit/303442ar

DOI : https://doi.org/10.7202/303442ar

Aller au sommaire du numéro

Éditeur(s)

Institut d'histoire de l'Amérique française

ISSN

0035-2357 (imprimé)

1492-1383 (numérique)

Découvrir la revue

Citer cet article

Simard, S. (1975). Les Français et le Canada, 1850-1914 : identité et perception. Revue d'histoire de l'Amérique française, 29(2), 209-239.

https://doi.org/10.7202/303442ar d'utilisation que vous pouvez consulter en ligne.

https://apropos.erudit.org/fr/usagers/politique-dutilisation/ 


\title{
LES FRANÇAIS ET LE CANADA, 1850-1914: IDENTITÉ ET PERCEPTION
}

\author{
Sylvain Simard \\ Montréal
}

Par leur nombre et leur diversité, les ouvrages publiés en France sur le Canada témoignent, à leur façon, de l'intensité des liens qui se sont créés et des relations qui se sont établies au cours de la seconde moitié du 19e siècle entre la France et son ancienne colonie. Ces ouvrages (près de 700 livres et brochures, voir tableau 1) ${ }^{1}$ intéressent l'historien à deux point de vue: ils constituent une importante source d'information sur le Canada au $19 \mathrm{e}$ siècle et ses relations avec l'extérieur; ils permettent aussi, par la meilleure connaissance de l'image du Canada transmise au public français, de juger de l'état de l'opinion en France sur le Canada.

Toutes ces publications n'ont évidemment pas la même importance et diffèrent autant par la qualité de leur contenu que par l'ampleur de leur diffusion. Une simple étude des tirages, rééditions et réimpressions permet de déterminer que les types d'ouvrages "canadiens" les plus largement diffusés en France sont, dans l'ordre, les récits de voyages, les romans d'aventures, les ouvrages de vulgarisation et les brochures de propagande en faveur de l'émigration. Les essais (études économiques, politiques, sociales, ethnographiques, géographiques, religieuses, etc...) atteignent évidemment un public spécialisé et donc restreint. ${ }^{2}$ Par ailleurs, l'importance de la part canadienne dans ces écrits varie considérablement: il y a toute une marge, par exemple, entre un récit de voyage de Xavier Marmier ${ }^{3}$ dont plus de la moitié du texte est consacrée à la description de son périple au Canada et les quelques pages canadiennes d'Edouard Rod ${ }^{4}$,

1 Nous ne tenons pas compte ici des articles parus dans les quotidiens, revues et périodiques.

2 L'importance relative des divers types d'écrits est la suivante: géographie, études sociales, religieuses, économiques et politiques, $39 \%$; histoire, $25 \%$; récits de voyage, $19 \%$; fiction, $17 \%$.

3 Xavier Marmier, Lettres sur l'Amérique (Paris, 1851), 2 vol. $119 \mathrm{p}$.

${ }^{4}$ Edouard Rod, Reflets d'Amérique, Petite collection (Paris, 1905),

[209]

RHAF, vol. 29, no 2 (septembre 1975) 
entre La colonisation en Nouvelle-France de Salone ${ }^{5}$ et la biographie de Tardivel par Mgr Fèvre. ${ }^{6}$ Il ne convient cependant pas de faire des distinctions entre ces diverses publications puisque la perception du Canada s'est formée en France grâce à l'ensemble de ces écrits, chacun d'eux apportant une touche complémentaire au portrait que le public lecteur a pu se créer. ${ }^{7}$

\section{TABLEAU 1}

Tableau décennal des publications (livres et brochures)

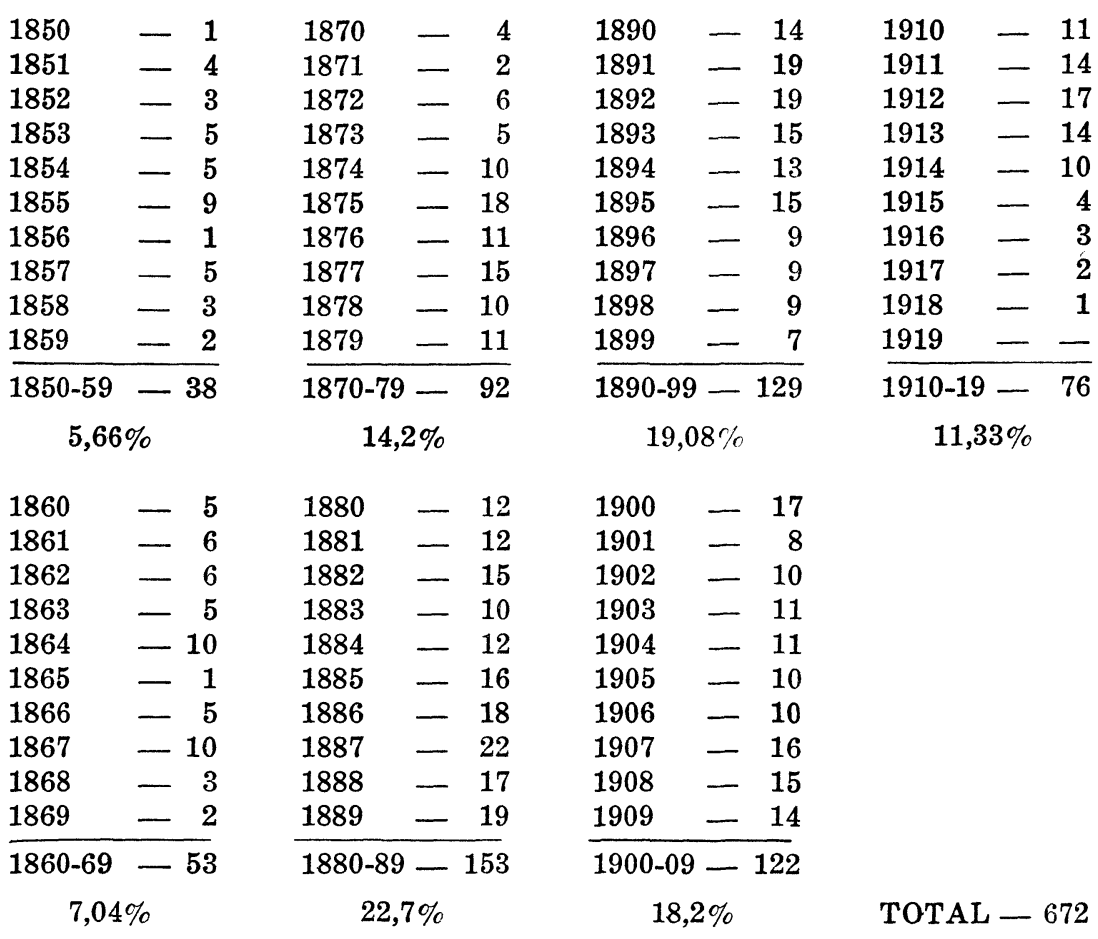

5 Emile Salone, La colonisation en Nouvelle-France - Etude sur les origines de la nation canadienne-française - Coll. lib. orientale et américaine (Paris, 1905), xii-467 p.

6 Mgr Justin Fèvre, Vie et travaux de J.-P. Tardivel, fondateur du journal La Vérité à Québec (Paris, 1906), $245 \mathrm{p}$.

7 Nous avons publié une partie importante de la bibliographie des écrits français sur le Canada, dans le 3e Cahier du Centre culturel canadien, "Les relations France-Canada au XIXe siècle" (Paris, 1975), 118 p. 
C'est afin de connaître le mieux possible ce portrait du Canada en France que nous nous intéressons depuis quelques années à ces écrits français sur le Canada. Nous avons rapidement compris, à travers nos lectures, que les façons diverses d'aborder dans ces livres la réalité canadienne sont étroitement liées à l'appartenance des auteurs à des groupes idéologiques déterminés, cette appartenance étant elle-même liée au milieu socio-économique des auteurs. Afin de vérifier cette intuition, il convient, dans un premier temps, d'identifier les grandes orientations idéologiques et l'appartenance socio-économique des auteurs, d'analyser ensuite les lignes de force du contenu des livres et brochures et d'établir enfin un certain nombre de corrélations entre l'identité et la perception. Nous laissons de côté dans cette enquête tout aspect évolutif, les limites d'un article nous forçant à nous en tenir au seul plan synchronique.

\section{Méthodologie}

Afin de connaître avec une certaine précision ceux qui en France se sont suffisamment intéressés au Canada pour écrire à son sujet et ce que contiennent ces écrits, nous avons procédé par sondage. A cette fin nous avons fait une enquête minutieuse à l'aide d'un questionnaire comprenant 112 interrogations. Ce questionnaire, nous l'avons appliqué à un échantillonnage de 100 ouvrages, soit un peu plus de $14 \%$ de l'ensemble du corpus. $\mathrm{Si}$ nous avons entrepris cette étude systématique, c'est qu'elle nous paraissait plus susceptible que l'étude individuelle de 700 cas d'établir les lignes de force de l'orientation de ces écrits. Nous n'attribuons évidemment pas aux différentes structures que nous avons fait émerger à l'aide de cette enquête un déterminisme absolu qui expliquerait clairement et totalement l'ensemble des cas individuels. Nous nous refusons cependant à ne voir, dans l'ensemble des écrits que nous avons étudiés, qu'une simple accumulation de faits de hasard.

Une première série de questions que nous appelons "questions d'identification" s'adressent à la personne des auteurs des écrits. Elle vise à identifier, de la façon la plus exacte possible, les groupes sociaux, politiques, économiques, religieux et culturels auxquels les auteurs appartiennent. La deuxième série de questions vise à découvrir les grandes lignes du contenu des œuvres: des centaines de sujets ont été abordés, mais il était important d'identifier et de comprendre les thèmes les plus souvent exploités. 
Que nous ayons retenu plus de $14 \%$ des titres du corpus constitue une assurance suffisante d'arriver à des conclusions qui puissent s'appliquer à peu près parfaitement à l'ensemble. Un sondage, aussi sérieusement conduit soit-il, demeure un sondage, avec ses limites et ses possibilités de distorsion. Si nous précisons que les informations que nous obtiendrons et les conclusions que nous en tirerons s'appliquent avant tout à l'échantillonnage interrogé et que des possibilités de marges d'erreurs existent, de telles possibilités, à cause de l'ampleur de l'échantillonnage, nous apparaissent peu probables.

Réunis selon les règles du hasard, les titres obéissent à certains impératifs. Les quotas ont été fixés afin de: $1^{\circ}$ suivre le plus fidèlement possible la distribution chronologique des publications ${ }^{8} ; 2^{\circ}$ correspondre à la classification des écrits selon les genres littéraires et les sujets abordés ${ }^{9}$.

Nous aurions pu limiter notre enquête aux auteurs sur lesquels nous possédions un certain nombre d'informations bibliographiques. Cela eut cependant faussé sensiblement la représentativité de notre échantillonnage. Les auteurs retenus par les instruments classiques de recherches biographiques ${ }^{10}$ appartiennent à ce que certains sociologues appellent un "champ de production culturelle reconnu", c'est-à-dire reflétant les préoccupations de l'élite dominante. Des auteurs dont l'histoire n'a pas retenu les noms, qui ont vécu hors du milieu culturel officiel, auraient peut-être été ainsi éliminés. Nous avons préféré l'absence de réponses à certaines questions d'identification au risque de fausser la représentativité de l'échantillonnage.

8 Nous avons voulu ainsi éviter que certaines périodes soient sur ou sous-représentées:

$\begin{array}{rr}1850-1859: & 5,66 \% \\ 1860-1869: & 7,04 \% \\ 1870-1879: & 14,2 \% \\ 1880-1889: & 22,7 \%\end{array}$

1890-1899: $19,08 \%$

$1900-1909: 18,2 \%$

$1910-1919: 11,33 \%$

9 Récits de voyages: 18,9\%; ouvrages de fiction: 17\%; essais (économie-politique-société-ethnographie-religion) : $11,66 \%$; monographies historiques: $11,3 \%$; ouvrages de vulgarisation: $6,99 \%$; histoire religieuse: $5,43 \%$; langue et littérature: $4,47 \%$; présence française dans le monde et au Canada: $4,31 \%$; géographie physique: $4,31 \%$; synthèse et vulgarisation historique: $4,17 \%$; émigration et colonisation: $4,17 \%$; textes anciens et rééditions: $3,87 \%$; relations France-Canada: 2,68\%. Ces catégories sont nécessairement imprécises et arbitraires et un ouvrage peut appartenir à plusieurs de ces catégories à la fois. Certains romans sont des récits de voyages à peine déguisés; des récits de voyages contiennent souvent de larges parties de synthèse historique, etc.

10 Nous nous en sommes tenus aux renseignements imprimés: dictionnaires, encyclopédies, etc. 
Certaines questions sur des thèmes importants ne s'adressent nécessairement qu'à une partie des ouvrages retenus. Il est peu probable qu'une étude sur les voies de communication au Canada contiennent des renseignements précis sur le clergé canadien. Dans chaque cas, la valeur de nos résultats sera fonction de la proportion de répondants. Certaines questions, par ailleurs, posent, à cause de leur formulation, la rareté, la discrétion et l'imprécision des sources biographiques, des problèmes délicats. Ainsi en est-il des questions concernant les positions religieuses et politiques des auteurs. Il est habituellement facile de situer ceux qui ont des opinions extrêmes; ultramontains et anticléricaux, par exemple, n'hésitent d'ordinaire pas à se définir dans leurs écrits et par leurs actes. Dans d'autres cas, la profession exercée nous permet de les situer avec assez de précision; prêtres, hommes politiques et journalistes peuvent être assez facilement identifiés par le groupe ou le parti auxquels ils appartiennent, le journal où ils écrivent. Lorsque les choses ne sont pas si simples, il nous a fallu poser, à partir d'indices, un jugement nécessairement faillible.

Les questions sont souvent fermées, les possibilités de réponses limitées et préétablies. La réalité des choses, il n'est pas inutile de le rappeler, n'obéit jamais à des catégories aussi simples, pour ne pas dire aussi simplistes. Dans les divers domaines qui touchent au comportement des individus, leurs idées et même leur situation économique, personne n'est réductible à des catégories aussi simples que "républicain de gauche", "catholique conservateur", et à des jugements aussi tranchés que "favorable", "mitigé" ou "défavorable". Si notre questionnaire utilise souvent ces divisions sommaires, c'est qu'il nous apparaissait avant tout nécessaire de dégager des lignes de force, à l'aide de données quantifiables statistiquement, quitte à raffiner notre analyse par la multiplicité des questions.

Afin d'obvier en partie aux inconvénients causés par l'utilisation du traitement statistique des données qui fait perdre de vue les caractéristiques individuelles et ne permet pas de les mettre en corrélation, ce qui est notre objectif, nous avons utilisé la méthode du fichier-image. Mise au point par des chercheurs de l'Ecole Pratique des Hautes-Etudes, cette méthode de traitement graphique de l'information consiste à rapporter sur la tranche d'une étroite fiche de carton, la valeur des diverses variables retenues, en utilisant pour ce faire un trait noir, plus ou moins long. Lorsque l'ensemble du fichier est constitué, on procède à divers classements qui font apparaître certaines configu- 
rations graphiques: ce sont là les corrélations. Si plusieurs des 20 manipulations que nous avons effectuées sur le fichier ne se sont pas avérées fructueuses, d'autres, par contre, nous ont permis de tracer les divers portraits-robots des auteurs et des écrits analysés. ${ }^{11}$

\section{I - LES AUTEURS}

\section{a) Identité des auteurs français qui ont écrit sur le Canada}

La grille socio-professionnelle utilisée dans l'établissement de ce tableau 2 peut prêter à confusion. Ainsi, le nombre de réponses est presque le double (151) du nombre d'auteurs que nous avons pu identifier. Un certain nombre d'auteurs retenus dans l'échantillonnage ont une situation socio-professionnelle qui recouvre plus d'un élément de cette grille. Il s'agit moins de déterminer avec précision la profession ou le métier exercé par chacun des auteurs, que de déterminer, à l'aide du type d'emploi, sa position approximative dans la hiérarchie sociale et professionnelle.

Certaines catégories socio-professionnelles très importantes sont absentes de ce tableau. Nous n'avons en effet rencontré, dans l'échantillonnage, aucun ouvrier ${ }^{12} \mathrm{ni}$ artisan qui ait écrit sur le Canada. Il est cependant possible, pour les raisons documentaires que nous avons mentionnées plus haut, qu'il se trouve un certain nombre d'employés, commis, voyageurs de commerce etc..., parmi les $14 \%$ d'auteurs sur lesquels nous ne possédons pas de renseignements biographiques. Le seul paysan rencontré est Louis Viel, fermier à Saint-Laurent au Manitoba. Encore que ce Français émigré, qui raconte à ses compatriotes les difficultés et les avantages de l'émigration dans les Plaines de l'Ouest cana-

11 Nous tenons à remercier M. Jean-Claude Robert, professeur à l'Université du Québec à Montréal, qui nous a initié à la technique du fichier-image. Il est techniquement impossible de reproduire ici ces graphiques.

12 Les seuls témoignages d'ouvriers, identifiés comme tels, rencontrés dans notre recherche, se limitent au rapport rédigé par un groupe d'ouvriers visitant Montréal et Québec au retour de l'Exposition internationale de Saint-Louis en 1904 où ils avaient été délégués par les syndicats français. Nous $\mathrm{y}$ apprenons, entre autres, que ces syndicalistes, socialistes et anarchistes, ont pris la parole à des meetings à Montréal et Québec devant des auditoires nombreux, qu'ils ont tenu des propos anticléricaux et lancé des mots d'ordre sur la solidarité prolétarienne et qu'ils ont été chaudement applaudis par leurs confrères canadiens qui ont chanté l'Internationale. Voir Albert Metin, Exposition Internationale de Saint-Louis, 1904. Délégation ouvrière française au Canada et aux Etats-Unis... (Paris, 1907), $\mathrm{x} \nabla-301 \mathrm{p}$. 
dien, n'est que le coauteur de ce livre ${ }^{13}$ (informateur) dont la rédaction est due à un professeur parisien, M. Léopold Leau ${ }^{14}$.

Ce qui ressort de ce tableau c'est l'abondance des auteurs exerçant une profession culturelle. Ecrivains, journalistes, professeurs, bibliothécaires, géographes, hommes de sciences constituent les $2 / 3$ des auteurs recensés. Cette proportion est cependant sensiblement diminuée si nous défalquons du total les doubles réponses, c'est-à-dire si nous éliminons les écrivains qui sont aussi professeurs ou bibliothécaires. Il n'en reste pas moins alors la moitié des auteurs, ce qui nous permet déjà de conclure à la part importante de la "culture" au niveau des écrits français sur le Canada et à celui de l'ensemble des relations franco-canadiennes. Cette première conclusion, pour importante qu'elle soit, ne doit pas nous étonner puisque les agents de production culturelle sont, par définition, ceux qui sont les plus susceptibles d'écrire des livres...

On remarque par ailleurs que la représentation des membres du clergé, quoique importante, ne dépasse pas, à première vue, la représentation du clergé à l'intérieur du milieu "intellectuel" français. Donc, abondance de prêtres et d'évêques à s'intéresser au Canada mais non sur-représentation flagrante, contrairement à ce qu'aurait pu nous inciter à croire la très grande cléricalisation du Québec. Les hommes politiques sont assez nombreux, mais cette catégorie inclut tous ceux qui exercent des responsabilités politiques de même que ceux qui font de la "politique active". Ce sont les capitalistes, commerçants, industriels, hommes d'affaires qui semblent les plus nettement sous-représentés par rapport à leur nombre dans la bourgeoisie française. Même si l'on sait que cette catégorie professionnelle a peu tendance à écrire des livres, on pourrait croire que l'aspect économique des relations franco-canadiennes a été peu développé. Il faut tempérer cette conclusion par le fait que plusieurs ouvrages d'ordre technique et économique ont été écrits par des publicistes à l'emploi ou à la solde d'entreprises capitalistes ou d'associations industrielles et commerciales. Le rapport du publiciste Edouard Agostini ${ }^{15}$ sur l'agriculture, le commerce et l'industrie au Canada a été préparé et rédigé à la demande et grâce à la commandite du Syndicat maritime et fluvial de France; les ouvrages sur le Canada

13 Louis et Jean, L'aisance qui vient (Paris, Bloud, s.d.).

14 Léopold Leau est le fondateur de l'association La Canadienne qui milite en faveur de l'envoi d'émigrants et de capitaux français au Canada.

15 Edouard Agostini, Agriculture, Industrie, Commerce. La France et le Canada. Rapport du Syndicat maritime et fluvial de France (Pau, 1886), $107 \mathrm{p}$. 
TABLEAU 2

Situation socio-professionnelle des auteurs

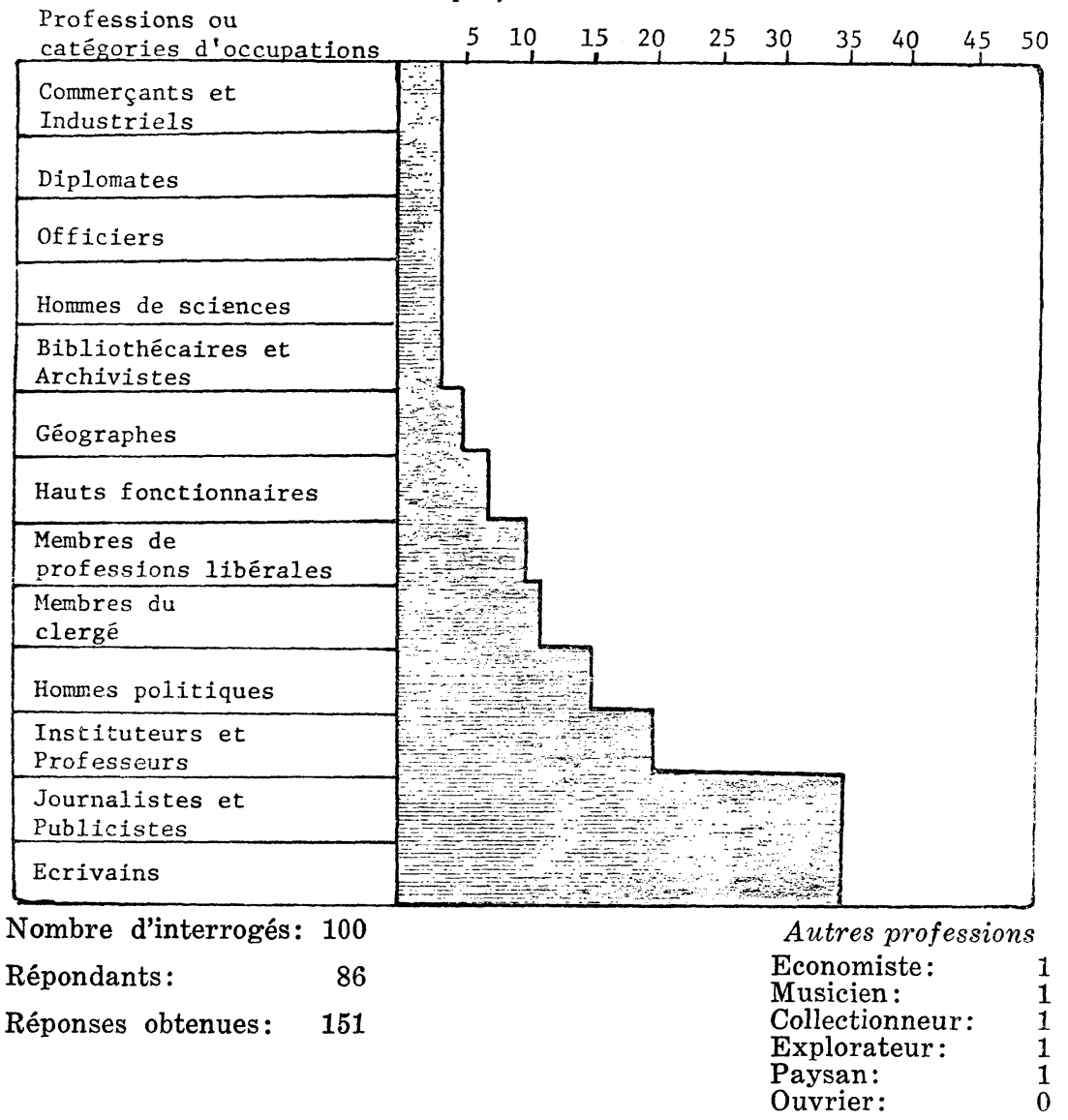

dus au journaliste et économiste Gustave de Molinari s'expliquent en partie par les liens étroits qui unissent l'auteur au groupe du Crédit foncier qui cherche à s'implanter et s'implantera effectivement au Canada.

Il convient aussi de souligner la présence parmi ceux dont la profession n'est pas identifiée, de plusieurs "fils de famille" et de "riches désœuvrés" venus au Canada dans l'espoir de se faire une situation ou simplement de s'instruire et de se former par le voyage. Maurice Barrès, par exemple, écrit en 1913 qu'il 
souhaite que le prince de Beauvau-Craon qui appartient à cette dernière catégorie, revienne en Lorraine "dans cette vaste plaine solitaire de Sion, où le plus beau château intact l'attend" et qu'il se mette au travail afin de réveiller son domaine et de collaborer à la renaissance de sa province:

Mais lui, écrit Barrès avec un peu d'agacement et d'amertume, il ne croit jamais s'être assez préparé, il ajourne à notre grand regret, l'heure d'un solide retour sur cette terre qu'il aime, dont il porte le nom, et hier encore, il s'en allait au Canada étudier, admirer, aimer des mœurs et des vertus de continuité auxquelles nous voudrions qu'il obéit lui-même en venant se dépenser au milieu de ceux qui toujours ont entouré sa famille. ${ }^{16}$

Cependant, même s'ils appartiennent à des couches sociales relativement élevées, la plupart des auteurs d'ouvrages "canadiens" doivent presque tous gagner leur vie. Ces nouvelles élites (professeurs - journalistes - instituteurs - hauts fonctionnaires) jouent un rôle de plus en plus important sous la IIIe République. De par leur situation professionnelle et leur position sociale, plusieurs d'entre eux sont à même d'exercer une profonde influence sur leurs compatriotes.

Le rayonnement d'un auteur et son degré de conservatisme sont souvent en relation étroite avec l'âge qu'il a atteint. Si près de la moitié des auteurs ont moins de quarante ans, rares sont ceux qui n'ont pas trente ans. Un examen attentif permet de constater que plusieurs d'entre eux sont au début de leur carrière. Henri de LaMothe n'est encore qu'un journaliste assez obscur lorsqu'il publie la relation de ses Cinq mois chez les Français d'Amérique, au Canada et à la Rivière Rouge; il deviendra, quelques années plus tard, un des principaux administrateurs coloniaux de la IIIe République. Remy de Gourmont n'est que bibliothécaire à la Nationale et n'a encore rien publié d'important lorsqu'il écrit ses deux ouvrages de commande sur le Canada. De nombreux auteurs atteignirent ainsi à une certaine renommée longtemps après s'être intéressés au Canada.

Plusieurs sont cependant d'ores et déjà connus et même, dans certains cas, célèbres. Qu'il suffise de citer Paul Bert, le comte Melchior de Vogüe, le contre-amiral de Cuverville, Gabriel Hanotaux, André Siegfried, Xavier Marmier, Jules Verne, Léon de Tinseau, Jean-Jacques Ampère, René Bazin, Maurice Barrès, Gustave Aimard, Alphonse Allais, le cardinal Touchet, Pierre

16 Maurice Barrès dans la préface à La survivance française au Canada du Prince de Beauvau-Craon (Paris, 1914), xxviii-235 p. 
Termier, Edouard Rod, le comte de Paris, Gustave de Molinari, Pierre Maël, Henri Lorin, l'abbé Félix Klein, le comte de Gobineau qui, à des titres divers, et ils ne sont pas les seuls, ont atteint une certaine renommée lorsqu'ils se préoccupent du Canada. Si la plupart d'entre eux sont aujourd'hui oubliés (qui aujourd'hui lit Barrès, Bourget ou Bazin?), certains jouirent alors d'une réputation considérable qui a certes influencé le rayonnement de ce qu'ils ont écrit sur le Canada et ses habitants.

Evidemment, pour un Bazin, un Siegfried ou un Allais, on retrouve dans la liste des auteurs plusieurs Gaillardet, Deville, Lamy, Bourbonneau, Quesnel et Deschamps, c'est-à-dire des auteurs qui, sans tous être dépourvus de talent, ont un rayonnement personnel très limité. Si la plupart des écrivains n'ont pas été choyés par la gloire, il faut se rendre compte que l'image d'un pays n'est jamais véhiculé dans le grand public par les seuls "grands écrivains".

L'âge relativement jeune d'une proportion importante des écrivains indique assez bien la nouveauté, surtout entre 1850 et 1885, de l'intérêt pour le Canada, les jeunes générations étant souvent les premières à s'intéresser à de nouvelles réalités. On assiste d'ailleurs à un vieillissement à la fin de la période considérée; on retrouve, entre 1900 et 1914, un plus grand nombre de quinquagénaires et de sexagénaires que dans la période précédente, ce qui indique un faible taux de renouvellement des auteurs. Si René Bazin n'a que 21 ans en 1884 lorsqu'il fait publier $M a$ tante Giron ${ }^{17}$, livre dans lequel les Canadiens sont assimilés à des planteurs louisiannais avec plantations et esclaves noirs, il en a 56 lorsque apparaît, en 1913, La Douce France, ouvrage ultra-nationaliste sur les provinces perdues et qui contient deux chapitres très significatifs sur le Canada. Onésime Reclus, qui a écrit ses premiers articles sur le Canada en 1863, à l'instigation de Rameau, continue de s'y intéresser pendant quarante ans ${ }^{18}$.

17 Entre-temps il a publié Nord-Sud, Amérique, Angleterre, Corse, Spitzberg où il fait une place importante au récit de son passage au Canada. En 1912, il fait partie de la Mission Champlain, en compagnie entre autres de G. Hanotaux, Etienne Lamy, Louis Blériot. Voir le récit de ce voyage dans la Revue des Deux-Mondes, "Paysages d'Amérique" (1er septembre 1912) : 49-87.

18 Le dernier ouvrage de Reclus où il est question du Canada porte le titre assez étonnant de Lâchons l'Asie, prenons l'Afrique, daté de 1904. Assez paradoxalement, cet apôtre de l'émigration française au Canada y invite les Canadiens à venir aider les Français dans leur colonisation africaine. Il semble qu'après la mort de Labelle en 1890 et l'effondrement des divers projets de colonisation que soutenait le "roi du Nord", Reclus ait un peu perdu foi aux possibilités mirifiques qu'il entrevoyait auparavant pour la race française en Amérique. 
Les auteurs qui écrivent un premier livre ou un premier article sur le Canada continuent très souvent de publier sur ce sujet pendant plusieurs années. Si tous n'ont pas, comme Onésime Reclus, Jean Lionnet, Henri-Emile Chevalier, Rameau et quelques autres, consacré l'essentiel de leur œuvre et de leurs efforts à faire connaître ce pays, ils sont nombreux ceux qui, tels $X$. Marmier, Th. Pavie, le baron Hulot, l'abbé F. Klein, L. Herbette, n'ont cessé de s'y intéresser et d'en faire le sujet d'études et de présentations. On peut parler, à propos de la majorité de ceux qui ont écrit sur le Canada, d'une véritable fidélité, et ce, même lorsque le contexte politico-religieux rendra difficiles et tendus les échanges culturels et intellectuels entre les deux côtés de l'Atlantique.

\section{b) La religion des auteurs}

La première constatation qui s'impose au sujet de la religion des auteurs qui ont écrit sur le Canada (Tableau 3), c'est la présence massive, parmi eux des catholiques ${ }^{19}$ et, par conséquent, la faible représentation relative des non-catholiques. S'il est vrai que la France de 1850 à 1914 reste un pays encore très imprégné par le catholicisme et que l'on a peut-être trop laissé dans l'ombre cet aspect de la société française, il n'en demeure pas moins que la représentation des catholiques parmi nos auteurs est nettement supérieure à ce que l'on connaît de la situation religieuse dans les couches supérieures de la société française auxquelles tous ces auteurs appartiennent ${ }^{20}$. L'instauration en France d'une République neutre, laïque et de plus en plus anticléricale, n'a pu se réaliser que grâce à la prépondérance de nouvelles élites non christianisées ou largement déchristianisées. Les auteurs qui ont écrit sur le Canada constituent donc, par rapport à l'ensemble de leur milieu social, un cas marginal; ils sont une minorité, nombreuse et active certes, mais une minorité.

Les catholiques ne forment cependant pas, dans la seconde moitié du $19 \mathrm{e}$ siècle, un groupe monolithique. Tant aux plans social, politique que religieux, l'Eglise de France connaît de nombreuses et profondes divisions. Le concile de Vatican I et l'infaillibilité pontificale, le toast d'Alger et le ralliement à la Répu-

${ }^{19}$ Nous entendons ici par catholiques ceux qui se définissent et s'affichent ainsi ou sont définis et considérés comme catholiques par leurs contemporains.

${ }_{20}$ Voir, à ce sujet, René Rémond, La Droite en France, de la 1ère Restauration à la Ve République. 
blique, l'affaire Dreyfus, - on pourrait citer une dizaine d'autres sujets - ont divisé profondément la conscience des catholiques français. La différence et l'antagonisme sont souvent plus grands entre un ultramontain légitimiste de l'Univers et un "libéral" orléaniste du Correspondant qu'entre catholiques et non-catholiques de mêmes tendances sociales et politiques. Ces divisions sur les rapports de l'Eglise et de l'Etat, sur le rôle de l'Eglise en éducation, sur le mode idéal de gouvernement et sur plusieurs autres sujets importants expliquent, en grande partie, une certaine impuissance politique du catholicisme français à la fin du $19 \mathrm{e}$ siècle.

TABLEAU 3

Religion des auteurs

Catholiques

"

,

, libéraux

conservateurs

ultramontains
28,3

31,0

9,7

Protestants

Les principales tendances qui coexistent plus ou moins pacifiquement au sein de l'Eglise de France se rencontrent aussi chez nos auteurs. Si le groupe majoritaire appartient aux tendances conservatrices, les catholiques libéraux sont cependant fortement représentés, alors que les ultramontains ne constituent même pas $10 \%$ des auteurs ${ }^{21}$. Connaissant l'influence prépondérante de cette dernière tendance au sein de l'Eglise canadienne, les relations suivies entre ultramontains français et canadiens, il est

21 Cela en surprendra sûrement plusieurs souvent enclins à croire que les relations France-Canada furent presqu'exclusivement le fait de fanatiques ultramontains. Leur influence dépassa peut-être leur importance numérique mais le phénomène ultramontain demeure une réalité marginale. On a souvent tendance à rappeler l'existence de Mgr Fèvre ou d'Ârthur Savaète tout en laissant dans l'ombre un "américaniste" comme l'abbé Klein ou un prêtre libéral comme l'abbé Lucien Lacroix. Les non-catholiques sont d'ailleurs absents, même chez les historiens contemporains, des relations France-Canada... 
assez surprenant de constater que, tout compte fait, les ultramontains aient été relativement peu nombreux à écrire des articles et ouvrages canadiens. Le radicalisme et la violence de leurs écrits, la force de certaines personnalités compenseront cependant en partie cette faiblesse d'effectifs. On peut en dire autant des écrits anticléricaux qui représentent un pourcentage semblable de l'ensemble des écrits.

En effet, sans tous être des anticléricaux militants, loin de là, $25 \%$ des auteurs peuvent être considérés comme agnostiques ou athées. L'image du Canada, surtout l'image religieuse, se ressentira donc de la présence de presqu'un tiers de non-catholiques parmi les auteurs. En cette période d'effervescence de la question religieuse qui constitue l'un des problèmes politiques primordiaux, l'appartenance des auteurs à différents groupes religieux, ou l'opposition d'une partie d'entre eux à l'Eglise et au clergé, influenceront fortement leur analyse de tous les secteurs de la vie canadienne.

Une des principales explications à la présence abondante des catholiques tient certainement au particularisme canadien luimême. Pays où les habitants d'origine française sont à peu près tous catholiques, dont l'idéologie est en grande partie définie par le clergé catholique, il est normal que le Canada ait surtout attiré l'intérêt de catholiques français. Intimement lié, nous le verrons, aux facteurs linguistiques, ethniques et sociaux, le facteur religieux joue un rôle majeur dans l'évaluation que l'on fait de la société canadienne. Très nombreux, parmi les auteurs qui ont écrit sur le Canada, sont ceux qui, désabusés ou irrités par l'impuissance des catholiques en France, sont venus chercher, dans ce pays, un exemple et un réconfort.

\section{c) Appartenance politique}

Il est difficile de comparer les résultats sur l'appartenance politique (Tableau 4) avec l'ensemble de la population française, puisqu'ils recouvrent plus de 60 ans de vie politique. Même si la majorité des auteurs ont écrit sous la République, celle-ci ne présente pas le même visage de l'Ordre moral à la République radicale de Combes ${ }^{22}$. Nous pouvons tout au plus remarquer que le pourcentage de monarchistes, tout en étant assez faible, est

22 Nous savons de plus que le conservatisme tend à se déplacer d'une position initiale d'anti-républicanisme à une participation conservatrice et affairiste. Voir à ce sujet Henri Guillemin, Nationalisme et Nationaux, $18 \% 0-1940$. 
TABLEAU 4

Appartenance politique des auteurs

Républicains

de droite

16,6

du centre

18,1

de gauche

24,2

Monarchistes

30,3

Bonapartistes

nettement supérieur au support obtenu par les partis dynastiques lors des élections de 1880 à 1914. Une autre lecture est possible; nous pouvons en effet opposer la droite conservatrice et la gauche radicale. Nous voyons alors que si nous assimilons les monarchistes à la droite, $50 \%$ de nos auteurs se classent nettement à droite de l'échiquier politique alors que nous n'avons pu en identifier que $18 \%$ à gauche. Cette nette tendance conservatrice est symétrique au tableau d'appartenance religieuse.

Nous pouvons donc conclure que la majorité des auteurs français ayant écrit sur le Canada, et ce, plus nettement à partir de 1880, s'opposent à la République radicale alors au pouvoir. Moins d'un auteur sur cinq, d'après ces tableaux, a pu applaudir aux lois Ferry ou aux lois Combes. En même temps que cela explique en partie leur intérêt pour ce Canada clérical et conservateur qui vit sous la Couronne britannique, cela pourra mieux faire comprendre l'origine et la nature de leurs prises de position, de certains jugements qu'ils porteront sur le Canada. Il serait cependant exagéré de voir le Canada comme la chasse gardée d'un groupe ultra-réactionnaire: ce pays a quand même intéressé des Français d'autres horizons politiques même si ceux-ci furent en nombre plus réduits que les premiers.

Un certain nombre de corrélations sont visibles au niveau de l'identification des auteurs. Ce qui apparaît le plus nettement, c'est la symétrie presque parfaite qui existe entre l'appartenance aux différents groupes religieux et les orientations politiques. Les catholiques libéraux sont, pour la plupart, républicains et se répartissent à peu près également entre les grandes tendances 
(gauche - centre - droite) ; les catholiques conservateurs sont quelquefois républicains de centre ou de droite mais, dans la très grande majorité des cas, monarchistes, comme le sont évidemment les ultramontains; les protestants, s'ils sont républicains, se rattachent au centre, alors que les agnostiques sont presque tous à gauche.

Il faut de plus constater que les auteurs catholiques appartiennent davantage à des sociétés savantes et à des sociétés de colonisation que les autres auteurs. Puisque l'appartenance aux sociétés savantes est surtout une réalité de province, nous pouvons voir là que la division religieuse est liée, du moins en partie, à la situation géographique. En fait, les auteurs anticléricaux sont presque tous de Paris. Le nombre relatif de pages consacrées aux Etats-Unis est plus élevé dans les ouvrages d'auteurs agnostiques que chez les catholiques. Il s'agit là d'un indice intéressant du très grand attrait de la république américaine pour les noncatholiques chez qui le Canada n'a pas le même intérêt.

Enfin, dernière corrélation, un lien unit l'appartenance religieuse et la position des auteurs dans l'échelle socio-professionnelle. En plus de la présence à laquelle il fallait s'attendre, de nombreux prêtres chez les catholiques, on retrouve chez eux, et presque exclusivement chez eux, les auteurs "sans profession", nobles désœuvrés, fils de famille, explorateurs amateurs, etc. Par contre, les hauts fonctionnaires et les hommes politiques catholiques sont peu nombreux.

En résumé, nous sommes devant une prépondérance de la représentation catholique parmi les auteurs d'ouvrages canadiens. Cette représentation catholique semble se caractériser par une tendance au conservatisme et se définir, jusqu'à un certain point, par une certaine impuissance politique à l'intérieur de la société française.

\section{II - GRANDES LIGNES DE LA PERCEPTION DU CANADA}

\section{a) La perception du Canada religieux}

La question religieuse est au cœur de la plupart des prises de position des auteurs français, comme elle est une composante majeure de l'opinion française sur le Canada. La France, pendant la seconde moitié du XIXe siècle, est secouée par d'importantes crises religieuses, crises qui atteindront leur paroxisme lors de l'affaire Dreyfus. C'est autour de la question religieuse, plus pré- 
cisément autour de la question cléricale, que se polarisent les conflits des divers mouvements d'idées et groupes d'intérêt qui divisent la société française. Problème latent sous le Second Empire qui mène une politique généralement favorable au clergé afin de se ménager l'appui de la Province, ce qu'il convient de nommer la "guerre religieuse" prendra son essor avec la Commune et surtout avec l'avènement de la République radicale qui succède à l'Ordre Moral. Il faut insister ici sur le climat passionnel et fondamentalement politique de toutes les prises de position sur la question religieuse. Il faut aussi souligner l'ambiguïté et l'inconfort où se trouvent plusieurs catholiques français, déchirés entre leurs convictions religieuses, leur libéralisme politique, les attitudes réactionnaires de la hiérarchie et l'intransigeance des milieux ultramontains. Si la lutte fait souvent rage entre radicaux et cléricaux, on doit garder à l'esprit que les catholiques sont eux-mêmes profondément divisés en plusieurs factions qui s'affrontent.

Les prises de position, les centres d'intérêt des auteurs sur la question cléricalo-religieuse au Canada sont presque toujours les échos fidèles de ces luttes françaises. Si certains auteurs réussissent à ne pas tirer de la situation religieuse canadienne des conclusions en relation directe avec leur perception de la situation française, il s'agit de cas exceptionnels. Aussi, la situation religieuse canadienne évoluant dans un sens diamétralement opposé à la situation religieuse en France, il ne faudra pas s'étonner qu'on ait, de part et d'autre de l'Atlantique, fait preuve d'une incompréhension croissante et qu'un fossé de plus en plus large se soit ainsi créé entre Français et Canadiens.

TABLEAU 5

La liberté religieuse existe-t-elle au Canada?

Nombre de répondants : 65

Nombre Pourcentage

1 - Existe

51

$78 \%$

2 - N'existe pas

14

$21 \%$ 
TABLEAU 6

Comment les auteurs jugent-ils la "richesse" du clergé?

Nombre total de répondants : 59

Nombre Pourcentage

1 - Tout à fait normale

47

$79 \%$

2 - Un peu exagérée

6

$10 \%$

3 - Choquante et injustifiée

6

$10 \%$

TABLEAU 7

Les pouvoirs socio-politiques du clergé devraient-ils être maintenus, diminués ou abolis?

Nombre total de répondants : 62

Nombre Pourcentage

1 - Maintenus

44

$70 \%$

2-Diminués

12

$19 \%$

3 - Abolis

6

$10 \%$

Les tableaux 5,6 et 7 concernent, à des degrés différents, les points essentiels du débat qui opposent, au $19 \mathrm{e}$ siècle, cléricaux et anticléricaux. S'ils ne constituent pas les véritables fondements des diverses prises de position, davantage d'ordre politique et économique, ils sont les thèmes d'opposition les plus souvent exprimés: pouvoir politique, biens du clergé, liberté religieuse. Nous retrouvons la matière des principaux débats, les prétextes des principales luttes qui secouent la société française. Ainsi que le démontre la très grande uniformité des réponses sur ces questions, les façons de percevoir chacun de ces sujets-clefs sont liées entre elles très intimement. Ceux qui affirment que la liberté religieuse existe au Québec peuvent difficilement critiquer les très grands pouvoirs et les propriétés du clergé. Ce qui est étonnant dans le très fort pourcentage d'approbation de ces divers points, c'est qu'il dépasse largement le pourcentage de catholiques qui n'est, rappelons-le, que de $68 \%$. 
Le Canada occupe une place privilégiée dans le cœur et dans l'esprit des Français pour des raisons qui sont d'abord fondamentalement des raisons sentimentales. L'analyse des diverses formes prises par cet attachement, d'autres motifs d'intérêt, des relations personnelles comme des tentatives de renouer concrètement certains liens, doit toujours tenir compte de l'importance primordiale de ce facteur sentimental, moteur principal des relations privilégiées avec un pays qui ne saurait autrement justifier un tel mouvement d'intérêt. Plusieurs pays sont catholiques et ne nous semblent pas prendre une place particulière dans le cœur des Français; ainsi en est-il de la structure sociale et politique et de l'économie. Ce qui attire a priori les Français, de quelque tendance religieuse ou politique qu'ils soient, c'est la réalité française du Canada. Les autres caractéristiques de la société canadienne détermineront la part plus grande prise par l'une ou l'autre de ces tendances sociales, politiques et religieuses dans ce mouvement. Ce qui nous permet d'affirmer que s'il eut été protestant ou anticlérical, le Canada eut quand même attiré l'attention et suscité l'intérêt de nombreux écrivains de l'Hexagone, mais que ceux-ci n'auraient pas été les mêmes.

On peut, s'il est nécessaire, trouver une autre preuve de la prépondérance de ce facteur dans l'intérêt presqu'exclusif des auteurs français envers les Canadiens francophones alors que, l'itinéraire emprunté par les voyageurs français le démontre bien, le Canada anglais laisse presque tous les Français complètement indifférents. Il ne faudra done pas se surprendre que l'image du Canada transmise à travers ces ouvrages soit essentiellement celle du Canada français. Ce n'est qu'accidentellement que l'on traite du Canada anglais et presque toujours en relation avec le Canada français.

Dans la lutte qui oppose à plusieurs reprises, pour des raisons linguistiques et "raciales", Canadiens francophones et Canadiens d'origine irlandaise, les Français seront souvent les plus virulents dans leurs attaques contre ces derniers, bien qu'ils soient catholiques. L'appartenance à une même religion, si elle demeure une cause importante de l'intérêt manifesté à l'égard des Canadiens, ne devient un facteur déterminant que lorsqu'elle est combinée à l'appartenance à une race et à une langue commune.

L'explication la plus plausible de ce phénomène est, croyonsnous, la primauté du facteur racial et linguistique sur la question religieuse chez plusieurs agnostiques. L'explication la plus souvent donnée, par les uns et par les autres, de cette position privi- 
légiée du clergé, est d'ordre historique. Ainsi, 63\% des auteurs invoquent cette explication: le clergé canadien, ayant joué et jouant encore un rôle de leadership patriotique depuis la défaite des armées françaises, il permet, de ce fait, la survivance et le développement de la nationalité française au Canada. Il est tout à fait normal, écrivent-ils, que les Canadiens lui soient reconnaissants et continuent de lui faire confiance. A ce facteur il convient d'ajouter que chez plusieurs anticléricaux socialement conservateurs le clergé constitue un élément important de la stabilité sociale.

Il n'y a que $20 \%$ des auteurs à considérer que le rôle du clergé ne fut peut-être pas déterminant pour la survivance de la race française en Amérique, et que, si tel était le cas, ce fait ne suffit pas à justifier la situation de pouvoir qu'il occupe. Mais le plus grand nombre adhère à la première thèse et cela pousse plusieurs non-catholiques à approuver, pour des raisons historiques et patriotiques, les structures religieuses canadiennes.

Ainsi le patriotisme français, l'anglophobie, la ferveur coloniale et le libéralisme économique se rejoignent chez quelques anticléricaux pour les faire se prononcer en faveur du maintien et même du développement de l'influence du clergé au Canada.

Le seul domaine où la proportion de points de vue critiques recouvre à peu près totalement celle des non-catholiques est celui qui concerne la position sociale et politique prédominante du clergé. En effet, près de $30 \%$ des ouvrages comportent une certaine critique du rôle joué par l'Eglise canadienne dans l'éducation: cette attitude part d'une critique du contenu jugé rétrograde de l'enseignement et surtout sur les interventions, estimées peu démocratiques, du clergé lors des élections. Ces questions, et surtout la dernière, ne font d'ailleurs pas l'unanimité au Canada où elles sont aussi l'objet d'âpres débats.

Les Canadiens ont adopté, dans leur majorité, le point de vue défini par le clergé. Les auteurs ont perçu, dans l'ensemble, l'ambiguïté de cette attitude des Canadiens à l'égard de la France. Si un véritable culte est rendu à la vieille France, celle de l'Ancien Régime, les Canadiens français sont très critiques à l'égard de la France du $19 \mathrm{e}$ siècle, surtout la France républicaine, celle de la Révolution, l'incarnation du mal. Cette attitude qui remonte d'ailleurs à la Révolution française ${ }^{23}$ fut renforcée dans les

${ }^{23}$ Le clergé canadien interprète la Conquête anglaise comme le moyen qu'a pris la Providence nour garder le Canada de la néfaste Révolution. 
TABLEAU 8

Le clergé suscite-t-il l'amour ou la haine de la France chez les Canadiens francais?

Nombre de répondants : 42

Nombre Pourcentage

1 - L'amour de la France contemporaine

2 - La haine de la France contemporaine

3 - L'amour de la France de l'Ancien Régime

4 - N'a joué aucun rôle

années " 60 " par la politique romaine de l'Empire, pour ensuite s'accentuer lors de la Commune, les lois Ferry et les lois Combes. Ces derniers événements donnèrent lieu à l'émigration de plusieurs communautés religieuses dont les membres ne manquèrent pas d'exciter ces sentiments antifrançais. S'il y a deux Frances pour les Canadiens, il y a aussi deux espèces de Français; ceux qui soutiennent la politique religieuse de leur pays et sont tous plus ou moins des suppôts de Satan et ceux qui s'opposent au nouvel ordre des choses et désirent un retour à l'ordre ancien. Ce sont ces derniers que l'on veut comme interlocuteurs puisqu'ils sont les seuls à représenter la "vraie France".

Ce qui, cependant, peut nous étonner, c'est que plusieurs auteurs français souscrivent à cette vision manichéenne de la société française. En effet, près de la moitié $(48 \%)$ des auteurs se disent en accord avec l'attitude des Canadiens face à leur pays. C'est là un signe assez clair de l'importance des conservateurs et ultra-conservateurs parmi ceux qui écrivent sur le Canada. Loin de se sentir ostracisés par le sentiment antifrançais de la majorité des Canadiens, ils y souscrivent et trouvent un appui à leurs propres prises de position. Ainsi, pour eux, non seulement le Canada incarne-t-il une société presqu'idéale, mais en plus, les Canadiens ont les mêmes objets de haine. Ils sont heureux de voir Jules Ferry objet de la même haine à Montréal que dans les pages de L'Univers. 
Parmi ceux qui, assez nombreux, contestent l'à-propos de cette vision de la réalité française, en plus des protestants et des anticléricaux, on retrouve plusieurs catholiques libéraux qui s'insurgent devant les simplifications et les caricatures que l'on fait d'une France qui serait globalement impie et débauchée et d'une République uniquement préoccupée à persécuter l'Eglise. Il y a même, parmi ces derniers, des prêtres qui tentent de convaincre les catholiques canadiens de la fausseté de cette image et essaient de faire comprendre aux Canadiens que, contrairement à ce que prétendent plusieurs membres de leur clergé, Paris n'est pas qu'un lieu de luxure et de débauche où l'on perd nécessairement son âme et qu'il y a encore des honnêtes gens en France. Ils incitent leurs collègues à plus de modération dans leurs jugements, comprenant bien qu'une telle attitude risque de compromettre toutes chances d'établir les relations nécessaires entre les deux peuples.

\section{b) L'infériorité économique des Canadiens français}

Pour les auteurs français le Canada n'est pas un pays complètement isolé. C'est en le comparant à d'autres pays, ses habitants à d'autre peuples, que plusieurs prennent position à son sujet. A l'intérieur du Canada même, les auteurs ont dû comparer la situation des Canadiens français avec celle des anglophones. Il n'y a que $51 \%$ des auteurs pour admettre qu'économiquement les Canadiens français sont dans une position d'infériorité si on les compare à leurs compatriotes de langue anglaise. Il est extrêmement révélateur de constater que $49 \%$ des auteurs nient cette réalité ou, au contraire, affirment que les Canadiens français occupent une position économique privilégiée au Canada. Tous les témoignages locaux et toutes les études entreprises depuis démontrent pourtant un phénomène qui n'a pas cessé d'être vrai, soit la disparité des revenus entre francophones et anglophones à l'avantage marqué pour ces derniers, soit l'état d'hygiène et de santé nettement inférieur du groupe francophone, soit la propriété presque exclusive des moyens de production non agricoles par les anglophones, de même que la domination sociale et politique de ces derniers, et ce, même à Montréal. Il fallait que le parti pris des auteurs envers les Canadiens français soit très fort pour que la moitié d'entre eux aillent jusqu'à nier cette évidence.

Parmi ceux que l'enthousiasme n'empêche pas d'admettre l'infériorité économique des Canadiens français, les causes avancées pour expliquer une telle situation ne font pas l'unanimité. Les raisons invoquées le plus souvent sont les suivantes. 


\section{TABLEAU 9}

Quelle est la cause de l'infériorité économique des Canadiens français?

Nombre total de répondants : 40

1 - Situation de dépendance coloniale du Canada français $40 \%$

2 - Instruction insuffisante ou mal orientée $26 \%$

3 - Infériorité raciale

(manque de dynamisme de la race française) $\quad 18 \%$

4 - Influence négative de la religion $16 \%$

Ces pourcentages, même s'ils ne concernent que quarante auteurs, viennent étayer certaines hypothèses que nous avons déjà proposées. Que la situation coloniale soit invoquée comme cause principale de l'infériorité économique ne doit pas nous étonner. Privés de l'apport économique, culturel et politique d'une mère patrie, les Canadiens français peuvent difficilement concurrencer à armes égales avec leurs compatriotes qui ont beaucoup reçu et reçoivent encore d'une Grande-Bretagne au faîte de sa puissance commerciale et industrielle. L'agriculture constitue le seul secteur où les Canadiens français continuent à jouer un rôle important puisqu'ils possèdent la majorité des terres cultivables de la province de Québec. Ces terres étant insuffisantes en nombre et en superficie, ils se voient d'ailleurs, tout au long de la seconde moitié du XIXe siècle, forcés de s'exiler dans les manufactures américaines ou dans les grandes villes québécoises, y formant le plus souvent un sous-prolétariat économiquement, socialement, politiquement et culturellement exploité.

A cette raison fondamentale se greffent d'autres raisons qui rallient l'opinion d'un nombre plus limité d'auteurs. Cependant, si on ajoute aux $16 \%$ de ceux qui rendent responsable de cette infériorité l'influence néfaste de la religion catholique les $26 \%$ de ceux qui attribuent celle-ci à la médiocrité et à la mauvaise orientation de l'éducation (l'enseignement relève presque complètement de l'initiative et du contrôle clérical), on s'aperçoit que, directement ou indirectement, l'idéologie dominante au Canada français est perçue comme un facteur important de la faiblesse économique. Aux anticléricaux se joignent les catholiques libéraux qui considèrent que l'orientation strictement huma- 
niste des études classiques que font les jeunes habitants de la province de Québec ne les prépare pas à lutter efficacement avec les jeunes anglophones dans le monde économique.

En ce siècle où les empires anglais et allemand se développent avec une telle rapidité et atteignent une domination économique et financière, il n'est pas rare de voir invoquer en France, et ce, souvent par des économistes et des hommes politiques renommés ${ }^{24}$, l'incapacité congénitale des Français, soit au développement économique, soit à l'expansion coloniale. Les difficultés rencontrées par la France dans son effort de colonisation, en Afrique et en Orient, ainsi que les diverses crises économiques traversées par un pays qui fait sa révolution industrielle près d'un siècle après celle de ses voisins, viennent accréditer cette théorie et ce fatalisme. Les Canadiens français, fondamentalement Français sous ce rapport, devraient, selon eux, à leur caractère latin, leur incapacité de concurrencer les Anglo-Saxons.

Certains auteurs utiliseront cet argument, parmi plusieurs autres, pour prétendre qu'ils doivent s'attacher uniquement à la culture du sol et laisser aux autres les activités considérées comme fondamentalement viles et immorales. Ainsi, selon eux, les Canadiens français doivent être fiers de leur infériorité: ils sont plusieurs à vanter "l'aurea mediocritas" du paysan canadien dont la mission sur cette terre est davantage spirituelle et culturelle qu'économique. D'ailleurs ce point de vue en détermine plusieurs à ne pas constater d'infériorité économique chez les Canadiens, puisque la vraie richesse n'est pas dans l'univers industriel et financier mais dans le travail du sol et l'acquisition des biens spirituels et culturels. Ce n'est donc pas parce qu'ils seraient incapables de se l'approprier que les Canadiens doivent laisser à d'autres la richesse matérielle; c'est qu'au contact du monde de l'usine ou des affaires, ils risquent de perdre ce qui fait leur force et leur valeur: leur langue et leur foi. Pour Rameau de Saint-Père comme pour Xavier Marmier ou Onésime Reclus, le salut des Canadiens réside dans la prise de possession du sol. Non seulement cela leur permettra de préserver, sous la direction du clergé, les valeurs fondamentales que sont la langue et la foi, mais leur permettra peu à peu une domination territoriale qui les rendra maîtres, grâce à leur démographie galopante, du territoire canadien.

24 Par exemple Edmond Demolins, A quoi tient la supériorité des Anglo-Saxons? 
Ils ne sont pas les seuls à ne voir l'avenir des Canadiens français que dans l'agriculture: en effet, si dans $31 \%$ des ouvrages, on trouve des remarques soulignant la nécessité qu'il y a pour le Canada de s'industrialiser, $14 \%$ des auteurs, par ailleurs, n'en voient pas du tout le besoin, alors que $10 \%$ s'y opposent tout à fait. Sans être majoritaires, nombreux sont les auteurs qui ont une vision essentiellement agriculturiste du présent et de l'avenir du Canada français. Il est difficile de savoir s'il s'agit de leur part d'une pétition de principes ou s'ils ne font que se soumettre à la réalité, soit la constatation de l'impossibilité pour les Canadiens français, dans le contexte colonial qui est leur, de penser réussir dans un domaine où les capitaux et les débouchés commerciaux leur sont difficilement accessibles. Font-ils de l'agriculturisme une doctrine idéale ou se plient-ils aux nécessités objectives? Seule l'étude détaillée de certaines œuvres peut permettre de faire la part de ces deux possibilités.

Cette volonté de ne pas ternir l'image du Canada en oubliant de mentionner certains de ses points faibles au plan économique est manifeste, puisqu'il n'y a que $26 \%$ des auteurs qui font allusion à l'émigration massive des Canadiens vers les cités industrielles américaines. Encore faut-il souligner que plusieurs d'entre eux y voient un signe de la vitalité expansionniste des Canadiens français qui s'emparent graduellement du contrôle de la partie nord des Etats-Unis! D'ailleurs peu d'auteurs, à peine $21 \%$, se penchent sur la situation économique des ouvriers canadiens, alors que les paysans retiennent l'attention de tous.

Cette vision d'un Canada avant tout agricole n'est évidemment pas uniquement d'ordre économique. C'est l'ensemble des mœurs d'une société rurale qui retient l'attention et suscite l'adhésion de la majorité des auteurs. Ainsi, si l'on établit la comparaison entre les Etats-Unis et le Canada, non seulement en termes socio-économiques, mais en termes de mœurs et de culture, on obtient des résultats révélateurs. Des 57 auteurs qui ont fait cette comparaison, il n'y en a que $10 \%$ à juger supérieures les mœurs et la culture des Américains, alors que tous les autres, à des degrés divers, les estiment inférieures. Les Américains sont peut-être plus riches, encore que la majorité des auteurs ne l'admettent pas, mais leurs mœurs sont viles, leur culture à peu près inexistante, en tout cas nettement inférieure à celle des Canadiens. Pour plusieurs des auteurs français qui écrivent sur le Canada, les Américains sont ces rustres Yankees incultes dont ils se plaisent à tracer un portrait très ironiaue, 
et ce, même si plusieurs de ces auteurs sont partisans du système social et politique américain. C'est, par contre, la civilité et l'hospitalité simple et chaleureuse des mœurs provinciales françaises, alliées au sens de l'ordre et du civisme des Anglo-Saxons, que les Français admirent chez les Canadiens.

\section{c) L'avenir politique du Canada}

Cet avenir du Canada, les auteurs le souhaitent ou l'envisagent de différentes façons. La plupart soulignent avec raison, surtout à la fin du XIXe siècle et au début du XXe siècle, que le lien colonial anglais est de plus en plus nominal, puisque les décisions importantes, y compris celles concernant la défense du pays, sont sous la juridiction exclusive du gouvernement canadien. Cette évolution n'était pas parvenue à ce stade au milieu du siècle et l'indépendance que souhaitaient ou prédisaient plusieurs auteurs ressemble beaucoup à la réalité de la fin du siècle.

\begin{tabular}{lc}
\hline \multicolumn{2}{c}{ TABLEAU 10 } \\
\multicolumn{2}{c}{ Quel est l'avenir politique du Canada? } \\
Nombre de répondants : 64 \\
$\begin{array}{lc}1 \text { - Rupture du lien colonial et indépendance du Canada } \\
2 \text { - Maintien du lien colonial }\end{array}$ & $41 \%$ \\
3 - Annexion aux Etats-Unis & $20 \%$ \\
4 - Fractionnement du Canada et indépendance & $17 \%$ \\
du Canada français (Province de Québec) & $6 \%$ \\
\hline - Retour du Canada à la France & $6 \%$ \\
\hline
\end{tabular}

C'est l'accession du Dominion canadien à la souveraineté complète qui apparaît, pour la majorité des auteurs, comme la solution la plus probable et la plus souhaitable. Cette accession progressive, inscrite dans les faits, constitue pour ceux-ci, le seul moyen d'échapper à la très forte attraction politique et économique de la République voisine qu'un éclatement de la Confédération rendrait inévitable. Pour plusieurs autres cependant, le Canada ne pourrait résister à lui seul aux formidables pressions expansionnistes des Etats américains. Aussi souhaitent-ils voir l'Angleterre continuer d'assumer sa tutelle sur le Canada qui jouit aussi de la protection de l'ensemble de l'Empire britannique. 
Parmi ceux qui préfèrent le maintien du statu quo se trouve un pourcentage non négligeable de royalistes pour qui la Couronne anglaise, digne héritière de la Couronne française, peut seule assurer aux Canadiens des institutions et un ordre social qui font de ce pays un Etat idéal. Plusieurs verraient d'un mauvais œil l'instauration d'une République canadienne, qui amènerait, selon eux, la répétition des erreurs dont sont victimes la France et les Etats-Unis. Ainsi, même parmi les plus nationalistes des auteurs, en trouve-t-on pour souhaiter que la Couronne anglaise puisse continuer à jouer un rôle important au Canada.

Les partisans de l'annexion aux Etats-Unis se retrouvent évidemment parmi les adversaires de la structure sociale canadienne où l'influence du clergé conservateur est primordiale et chez les admirateurs de la République américaine. D'autres, sans le souhaiter, constatent que la puissance économique et politique des Américains conduira tôt ou tard ces derniers à s'emparer de ce pays qui constitue le prolongement naturel du leur.

Il n'y a que $12 \%$ des auteurs qui désirent ou la constitution d'un Etat francophone indépendant ou le rattachement du Canada à la France. Il n'est pas étonnant que la solution indépendantiste au Canada soulève aussi peu d'enthousiasme: la faiblesse économique et politique des Canadiens français rend, à cette époque, une telle perspective bien peu plausible. Le Canada, ainsi fractionné, deviendrait une proie trop facile pour le voisin yankee, même si certains espèrent assister à une reconquête du Nord de l'Amérique par les francophones. Il n'y a que quelques ultranationalistes pour rêver d'un retour possible à la France; si certains d'entre eux, tel Emile Lonchampt, violemment anglophobe, incitent leurs compatriotes à songer à une reconquête militaire de la colonie perdue afin de venger Fachoda et les autres échecs de la France aux mains de l'Angleterre dans son entreprise coloniale, il est peu probable que cette solution ait provoqué autre chose qu'un soupir de regret ou un sourire de scepticisme chez leurs lecteurs, tellement une perspective semblable apparaît peu réaliste dans le contexte militaire et politique.

\section{CONCLUSION}

Il convient d'établir certaines corrélations entre ces orientations principales du contenu des écrits "canadianistes" et la typologie des auteurs que nous avons établie. 
La sympathie raciale et linguistique apparaît dès le départ comme l'élément principal de l'intérêt des Français pour le Canada. Ce facteur semble transcender dans la plupart des cas les différences idéologiques, mais non pas les abolir. Ces auteurs sont plus divisés en ce qui concerne la religion, le clergé, l'économie et la politique.

En fait, la question religieuse, intimement liée à la question politique, est au carrefour des mouvements d'opinion sur le Canada. C'est, à partir des prises de position sur la réalité religieuse, que se divisent les écrits et les réflexions sur les autres aspects de la réalité canadienne. Ceux qui désirent le maintien des pouvoirs sociaux et politiques du clergé sont presque unanimes à trouver normale la richesse relative du clergé canadien. Presque tous considèrent que la liberté religieuse existe sur les bords du St-Laurent, justifient par l'histoire la situation privilégiée du clergé et attribuent à celui-ci, nous l'avons vu, la première responsabilité dans le maintien et le développement de la race française. A ce premier groupe, nettement majoritaire, appartiennent surtout des catholiques conservateurs et ultramontains ainsi que plusieurs catholiques libéraux.

D'autres auteurs, moins nombreux, considèrent que les pouvoirs sociaux et politiques du clergé dans la société canadiennefrançaise sont nettement exagérés. Ces auteurs ont des opinions partagées sur les autres aspects de la réalité catholique canadienne, oscillant entre une approbation totale et une attitude critique modérée. A ce groupe appartiennent, en nombre à peu près égal, des catholiques libéraux, des protestants et des agnostiques.

Un dernier groupe d'auteurs, aussi homogène que le premier, nie l'existence d'une quelconque liberté religieuse au Canada, dénonce les pouvoirs et possessions du clergé canadien et n'attribue pas à ce dernier la responsabilité principale du maintien de la nationalité française en Amérique.

Carrefour, avons-nous dit, des divers mouvements d'opinions et jugements sur la réalité canadienne, la question religieuse, nettement liée à l'orientation politique, semble déterminer, ou du moins, aller de pair, avec une série d'attitudes sur des sujets tout à fait différents.

Ainsi, les membres du premier groupe que nous avons identifiés, ceux qui approuvent totalement la situation religieuse et cléricale canadienne, sont ceux qui, sur tous les sujets, font preuve 
de plus d'enthousiasme à l'égard du Canada, enthousiasme frôlant même souvent l'admiration aveugle. Les auteurs conservateurs se gardent à peu près tous d'émettre quelque critique que ce soit à l'égard de l'économie canadienne. Tout se passe comme si, pour ces auteurs, la réalité économique, peut-être parce qu'elle risquerait de relativiser leur admiration pour le rôle social et politique du clergé canadien, devait être passée sous silence.

Plusieurs catholiques libéraux ${ }^{25}$ et quelques agnostiques font état de cette infériorité économique des Canadiens français par rapport aux Canadiens anglais et des Canadiens par rapport aux Américains. Ils rendent la situation coloniale dans laquelle vit le Canada, responsable de cette infériorité.

Enfin, les radicaux, agnostiques et protestants de gauche, critiques inconditionnels de la religion et du clergé au Canada, imputent cette infériorité économique à l'influence néfaste de la religion et à la nette insuffisance quantitative et qualitative de l'éducation ${ }^{26}$. Cela n'empêche pas plusieurs auteurs de ces $2 \mathrm{e}$ et 3e groupes de croire que le Canada est un pays relativement riche, que les paysans y sont beaucoup plus prospères qu'en France et d'inviter les paysans français à émigrer au Canada.

Ainsi, les façons d'aborder les éléments majeurs de la réalité canadienne: la religion et le clergé, la nationalité française, la situation économique, la proximité des Etats-Unis, l'attitude des Canadiens face à la France, l'avenir politique, sont presque toujours étroitement liées à la personnalité des auteurs. Aux trois groupes que nous avons analysés correspondent des types de comportements spécifiques. L'image du Canada ainsi transmise est moulée aux caractéristiques principales des auteurs qui la forgent. Sans doute répond-elle de la même façon, à l'attente du public ou plutôt, des différents publics. Au public conservateur, on présente un Canada à sa mesure et correspondant à son attente; de façon moins vaste, les publics libéraux modérés et radicaux reçoivent eux aussi une image du Canada qui coïncide, sur tous les points, à leur vision du monde, à leur idéologie.

A partir d'appréciations opposées, les tenants des diverses idéologies trouvent au Canada la preuve de la justesse de leur vision du monde. Les conservateurs y trouvent un pays catholique aux mœurs traditionnelles où ils se sentent parfaitement à

25 Tous les républicains.

${ }^{26}$ Puisque l'éducation est entièrement, directement ou indirectement, sous la responsabilité du clergé, cette critique constitue une autre attaque contre le rôle que joue ce clergé. 
l'aise: l'union de leur idéal d'Ancien Régime et de progrès social et matériel. Les libéraux y voient l'exemple excellent de l'adaptation nécessaire au modernisme: un pays où l'Eglise et la démocratie font bon ménage. Les radicaux y découvrent partout la preuve de la totale déchéance sociale, morale et politique où mènent inévitablement le cléricalisme et l'obscurantisme religieux.

Si l'on excepte les ultramontains qui trouvent au Canada français un ordre de choses qui ne diffère pas tellement de leur idéal, et cela, même si certains maximalistes comme Mgr Fèvre ou Dom Paulatim considèrent que les pouvoirs du clergé sont encore insuffisants, il est quand même étonnant de constater une telle unanimité des catholiques à approuver la situation canadienne. Il faut remarquer que les motivations des catholiques conservateurs sont sensiblement différentes de celles de leurs coreligionnaires libéraux à ce sujet.

Pour les conservateurs, le Canada français, au point de vue religieux, mais pas seulement à ce point de vue, constitue un modèle de réussite. En France, les catholiques font difficilement entendre leurs voix: ils sont écartés des postes de commande du pouvoir politique et même de la haute fonction publique et de l'armée, dans un pays où l'influence de la francmaçonnerie augmente tous les jours, où l'éducation est de plus en plus laïcisée et où le recrutement des clercs est à son plus bas. Le Canada offre, en contraste, l'exemple d'un pays où les catholiques possèdent le haut du pavé dans tous ces domaines. Si certains regrettent que le pouvoir économique et les grandes décisions politiques leur échappent, tous sont en admiration devant cette société encore profondément rurale, ayant conservé les mœurs archaïques et simples des ancêtres, respectant avant tout Dieu, l'Eglise et le roi.

Dans une société française qui, depuis un siècle, connaît bouleversements après bouleversements, le Canada, pays de la dîme et d'un ordre social qui rappelle, sur certains points, l'Ancien Régime, exerce un attrait si puissant que certains conservateurs opèrent un véritable "transfer", ce pays réalisant leurs rêves et leurs aspirations, véritable paradis perdu épargné par le péché de la Révolution. Dans leur correspondance, certains d'entre eux, Xavier Marmier et Léon de Tinseau entre autres, affirment qu'en cas de dégradation de la situation en France plusieurs "bonnes familles" se préparent à émigrer au Canada. Pour la très grande majorité des catholiques qui s'intéressent à ce pays 
du Nouveau Monde, il apparaît comme un oasis, un hâvre de paix contre-révolutionnaire dans ces temps difficiles.

Les "libéraux" partagent dans l'ensemble cet enthousiasme, mais leurs raisons sont quelquefois différentes. Ce qu'admirent avant tout Albert Lefaivre, l'abbé Lucien Lacroix et plusieurs autres, c'est le leadership social exercé par le clergé dans un pays démocratique où toutes les libertés formelles sont reconnues. Ils insistent sur la capacité de l'Eglise, dans un contexte de démocratie et de séparation d'avec l'Etat, d'exercer une influence prépondérante dans la société. Voyez l'exemple canadien, disent-ils à leurs compatriotes, où l'Eglise n'a pas besoin d'une situation juridique privilégiée pour vivre et se développer. Cette Eglise qui doit tout à ses membres demeure plus près de ceux-ci et reflète davantage leurs préoccupations et leurs aspirations.

Il est cependant une minorité qui, même si elle ne dépasse sur aucun des points posés dans ces tableaux, plus de $20 \%$ de l'échantillonnage, conteste globalement et fortement le rôle de l'Eglise et de la religion au Canada. Du protestant Grandpierre au voltairien Foubert, du journaliste radical Jousselin à l'ardent propagandiste Paul-Théodore Vibert en passant par le romancier H.-Emile Chevalier, on retrouve toute une gamme d'attaques et de critiques contre la réalité canadienne. Alors que pour certains protestants, l'Eglise catholique répand l'ignorance et détruit les vertus sociales et est, de ce fait, responsable de l'infériorité économique et sociale des Français d'Ámérique, les libres penseurs voltairiens comme les radicaux et les socialistes s'attachent surtout à démontrer l'aspect néfaste de l'obscurantisme religieux, surtout en matière d'enseignement. Le tableau du Canada tracé par les anticléricaux est parfois d'une telle noirceur que leurs pamphlets en viennent à perdre toute crédibilité.

Nous retrouvons donc une correspondance étroite entre l'appréciation de la nature et des conséquences de la situation canadienne et les prises de positions antérieures, chacun semblant ne voir au Canada que ce qui sert à confirmer son analyse de la situation française.

Une telle étude, pour être tout à fait fidèle à la réalité, aurait dû tenir compte, si l'espace d'un article de revue l'avait permis, de l'évolution des situations des deux côtés de l'Atlantique entre 1850 et 1914 et suivre diachroniquement les diverses étapes de la constitution d'une image du Canada en France. Au cours de ces soixante années, la France traverse des régimes politiques 
différents et connaît sa Révolution industrielle. Le Canada de 1914 est sensiblement différent aux plans économique, politique et idéologique des colonies anglaises de 1850. En France, le laïcisme d'Etat et le républicanisme anticlérical sont surtout des réalités de la dernière partie de la période envisagée et ont influé sur les relations entre les deux pays symétriquement à la mainmise cléricalo-conservatrice au Canada. Ce nationalisme (la Commune) et l'expansionnisme français (Jules Ferry) d'abord incarnés par la gauche, sont par la suite assumés par la droite. La gauche radicale anticléricale, en tant que force d'appui du capitalisme libéral, est socialement conservatrice. Le portrait d'ensemble que nous avons tracé reste cependant valable dans le cadre d'interprétation statique que nous nous sommes imposé. Ce n'est qu'à partir d'un tel tableau qu'une analyse plus raffinée de la perception du Canada en France reste possible. 